\title{
Suboptimal Outcomes in Cutaneous Squamous Cell Cancer of the Head and Neck with Nodal Metastases
}

\author{
VAMSI VARRA $^{1}$, NEIL M. WOODY ${ }^{1}$, CHANDANA REDDY ${ }^{1}$, NIKHIL P. JOSHI ${ }^{1}$, JESSICA GEIGER ${ }^{2}$, \\ DAVID J. ADELSTEIN ${ }^{2}$, BRIAN B. BURKEY ${ }^{3}$, JOSEPH SCHARPF ${ }^{3}$, BRANDON PRENDES $^{3}$, ERIC D. LAMARRE ${ }^{3}$, \\ ROBERT LORENZ ${ }^{3}$, BRIAN GASTMAN ${ }^{4}$, BINDU V. MANYAM ${ }^{1}$ and SHLOMO A. KOYFMAN ${ }^{1}$ \\ Departments of ${ }^{1}$ Radiation Oncology, ${ }^{2}$ Solid Tumor Oncology, ${ }^{3}$ Otolaryngology and \\ ${ }^{4}$ Plastic Surgery, Cleveland Clinic, Cleveland, OH, U.S.A.
}

\begin{abstract}
Background/Aim: There are limited data regarding survival, failure patterns, and factors associated with disease recurrence in patients with cutaneous squamous cell cancer of the head and neck (cSCC-HN) with nodal metastases. Patients and Methods: A retrospective analysis of patients with cSCC-HN metastatic to cervical and/or parotid lymph nodes treated with surgery and post-operative radiation therapy was performed. Results: This study included 76 patients $(57$ immunocompetent and 18 immunosuppressed) with a median follow-up of 18 months. Overall survival, disease-free survival $(D F S)$, and disease recurrence $(D R)$ at 2 years was $60 \%$, $49 \%$, and $40 \%$, respectively. Immunosuppressed patients had significantly lower 2-year DFS (28\% vs. 55\%; $p=0.003)$ and higher DR $(61 \%$ vs. $34 \% ; \quad p=0.04)$ compared to immunocompetent patients. Analysis of immunocompetent patients demonstrated extracapsular extension $(E C E)$ as the only factor associated with DR $(p<0.0001)$. Conclusion: Patients with nodal metastases from cSCC-HN have suboptimal outcomes. ECE and immunosuppression were significantly associated with DR.
\end{abstract}

Cutaneous squamous cell carcinoma (cSCC) is the second most common skin cancer, with an estimated annual incidence of 700,000 cases in the United States (1). The majority of cSCC tends to carry a favorable prognosis with high rates of local control and rates of distant metastasis as low as $2-3 \%$ (2). Overall, $5-20 \%$ of patients present with nodal metastases from cSCC (3). While uncommon, the presence of nodal metastasis

Correspondence to: Shlomo Koyfman, MD, Department of Radiation Oncology \& Bioethics, Cleveland Clinic, 9500 Euclid Ave - CA50, Cleveland OH 44195, U.S.A. Tel: +1 2164447552, email: koyfmas@ccf.org

Key Words: Cutaneous squamous cell carcinoma; nodal metastases, head and neck, skin cancer, immunosuppression, extracapsular extension, extranodal extension. is associated with higher rates of locoregional recurrence (14$20 \%)$ and distant recurrence (10-25\%) (4). The Brigham \& Women's Hospital Staging System has identified primary tumor characteristics associated with worse outcomes, including tumor diameter $\geq 2 \mathrm{~cm}$, poorly differentiated histology, depth of tumor invasion beyond fat, and perineural invasion $\geq 0.1 \mathrm{~mm}$, in addition to other high risk features identified in the literature including, immunosuppressed status, head and neck location, and recurrent tumor (5-8). The American Joint Committee on Cancer (AJCC) 8th edition Staging system includes the number of involved lymph nodes and the size of lymph node metastasis as prognostic factors (9).

New staging systems have introduced increased granularity and stratification of primary tumor characteristics; however, the literature on characteristics and outcomes of nodal spread is limited. The current nodal staging system is borrowed from mucosal head and neck squamous carcinoma as there is a paucity of published data on how patients with cSCC with nodal involvement behave. Also, non-melanomatous skin cancers are not captured in national databases like the Surveillance Epidemiology and End Results (SEER) and National Cancer Data Base (NCDB). This study sought to identify factors associated with disease recurrence and report failure patterns and survival outcomes in patients with nodal metastases from cutaneous squamous cell cancer of the head and neck (cSCC-HN) treated with surgery and post-operative radiotherapy (RT).

\section{Patients and Methods}

This institutional review board-approved retrospective study included patients with cSCC-HN with metastasis to cervical and/or parotid lymph nodes, treated with surgery and post-operative RT between 2002 and 2017. Patients with TX-T4 primary tumors, primary or recurrent disease and any immune status were included. Immunosuppression was defined as treatment with any immunosuppressive agent for greater than 6 months, or 2 months in the case of cyclophosphamide, or patients with a diagnosis of chronic lymphocytic leukemia. Patients with distant or in-transit metastasis 
only and those treated with palliative RT doses were excluded. All patients received surgical resection by Mohs Micrographic surgery or wide local excision, and lymph node dissection with a parotidectomy as clinically indicated. All patients received post-operative RT to the tumor bed, lymph node bed, or both. Often, more extensive elective nodal regions were treated as well if considered to be at significant clinical risk. A small portion of the patients received concurrent chemotherapy at the discretion of the treating oncologist based on performance status and disease severity.

All patients were followed-up with post-operative treatment imaging with computed tomography or positron emission tomography 3 months after RT. Locoregional recurrence was defined as recurrence at the primary site, margin of primary site, or regional lymph nodes. Distant recurrence was defined as recurrence at distant lymph nodes or other organs. Disease-free survival was calculated from the date of diagnosis to the date of recurrence, date of death, or last oncologic follow up. Overall survival was calculated from the date of diagnosis to the date of death or last date known alive. Baseline characteristics were compared using Fischer's exact test for categorical variables and Mann-Whitney test for continuous variables. Kaplan-Meier method was used to calculate overall survival (OS) and disease-free survival (DFS) and cumulative incidence curves were generated for any disease recurrence (DR=any local, regional or distant failure). Univariate and multivariate analyses were performed using Cox proportional hazards regression to identify variables associated with DR. A $p$-value $\leq 0.05$ was considered statistically significant. R software was used for statistical analysis and the CMPRSK package in the $\mathrm{R}$ statistic software was used to calculate cumulative incidence of recurrence.

\section{Results}

Of the 76 patients included in this study, 57 (75\%) were immunocompetent, median age was 72 and median follow-up was 18 months (range $=1-203$ months). The most common $\mathrm{T}$ stage was $\mathrm{T} 0 / \mathrm{X}(70 \%)$ and most common $\mathrm{N}$ stage was $\mathrm{N} 2 \mathrm{~b}$ (59\%). Overall, $45 \%$ of patients had poorly differentiated tumors, $42 \%$ had perineural invasion, $29 \%$ had lympho-vascular space invasion. Extracapsular extension (ECE) was present in $82 \%$ and was similar in the immunocompetent and immunosuppressed cohorts. All patients received surgical resection and adjuvant radiotherapy (median dose $60 \mathrm{~Gy}$ ), while $14.5 \%$ received concurrent cisplatin or cetuximab (Table I).

Locoregional failure occurred in 18 patients $(24 \%)$ and distant failure occurred in $14(18 \%)$. Estimated two-year OS (Figure 1), DFS and disease recurrence rates (DR) were 60\%, $49 \%$ and $40 \%$, respectively (Figure 2). Immunosuppressed patients had significantly lower two-year DFS (28\% vs. 55\%; $p=0.003$ ) (Figure 3) and higher DR (61\% vs. 34\%; $p=0.04)$ compared to immunocompetent patients (Figure 4).

Univariate analysis showed that immunosuppressed status (hazard ratio [HR] 2.52; $p=0.034$ ) and use of chemotherapy $(\mathrm{HR}=2.825 ; p=0.005)$ were the only variables significantly associated with DR. On multivariate analysis, immunosuppression $(\mathrm{HR}=2.2 ; p=0.05)$ and the use of chemotherapy ( $\mathrm{HR}=2.7 ; p=0.02)$ remained significant (Table II). In a separate analysis including only immunocompetent
Table I. Patient, tumor, and treatment characteristics $(N=76)$.

\begin{tabular}{|c|c|}
\hline Variable & No. of patients $(\%)$ \\
\hline Male/Female & $68 / 8(90 / 10)$ \\
\hline Caucasian/Other & $73 / 3(96 / 4)$ \\
\hline \multicolumn{2}{|l|}{ Immunosuppressed } \\
\hline Yes & $57(75)$ \\
\hline No & $19(25)$ \\
\hline \multicolumn{2}{|l|}{ Tumor stage } \\
\hline $\mathrm{T} 1$ & $1(1)$ \\
\hline $\mathrm{T} 2$ & $13(17)$ \\
\hline $\mathrm{T} 4$ & $9(12)$ \\
\hline $\mathrm{T} 0 / \mathrm{X}$ & $53(70)$ \\
\hline \multicolumn{2}{|l|}{ Nodal stage } \\
\hline N1 & $21(28)$ \\
\hline $\mathrm{N} 2 \mathrm{a}$ & $8(11)$ \\
\hline $\mathrm{N} 2 \mathrm{~b}$ & $45(59)$ \\
\hline N3 & $2(3)$ \\
\hline \multicolumn{2}{|l|}{ Cell differentiation } \\
\hline Well & $6(8)$ \\
\hline Moderate & $33(43)$ \\
\hline Poor & $34(45)$ \\
\hline Unknown & $3(4)$ \\
\hline \multicolumn{2}{|l|}{ Margin status } \\
\hline Negative & $39(51)$ \\
\hline Positive & $10(13)$ \\
\hline Unknown & $2(3)$ \\
\hline N/A & $25(33)$ \\
\hline \multicolumn{2}{|l|}{ Perineural invasion } \\
\hline Negative & $39(51)$ \\
\hline Positive & $32(42)$ \\
\hline Unknown & $5(7)$ \\
\hline \multicolumn{2}{|l|}{ LVSI } \\
\hline No & $48(63)$ \\
\hline Yes & $22(29)$ \\
\hline Unknown & $6(8)$ \\
\hline \multicolumn{2}{|l|}{ ECE } \\
\hline No & $12(16)$ \\
\hline Yes & $62(82)$ \\
\hline Unknown & $2(3)$ \\
\hline \multicolumn{2}{|c|}{ Use of chemotherapy } \\
\hline No & $65(86)$ \\
\hline Yes & $11(15)$ \\
\hline \multicolumn{2}{|l|}{ Radiation type } \\
\hline Electrons & $4(5)$ \\
\hline IMRT & $47(62)$ \\
\hline Type unknown & $25(33)$ \\
\hline
\end{tabular}

patients $(\mathrm{N}=57)$, the presence of ECE was most strongly associated with increased DR $(p<0.0001)$ in addition to the use of chemotherapy (HR=5.16; $p=0003$ ) (Table III). No immunocompetent patient failed in the absence of ECE.

\section{Discussion}

The literature characterizing nodal prognostic factors for cSCC-HN is limited. While Karia et al. analyzed 1,818 cases 


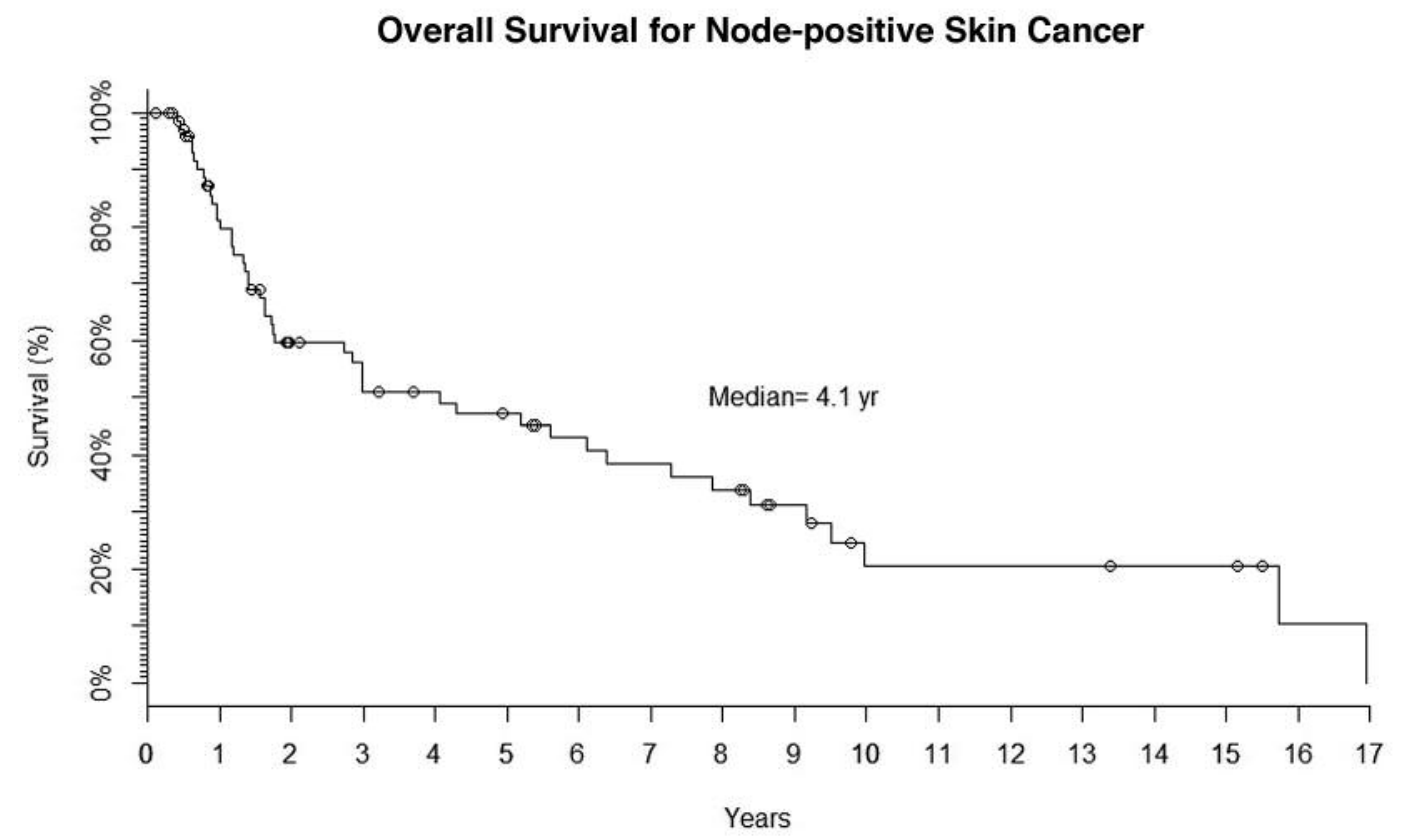

Figure 1. Overall survival of 76 patients with node-positive skin cancer.

\section{Disease Recurrence for Node-positive Skin Cancer}

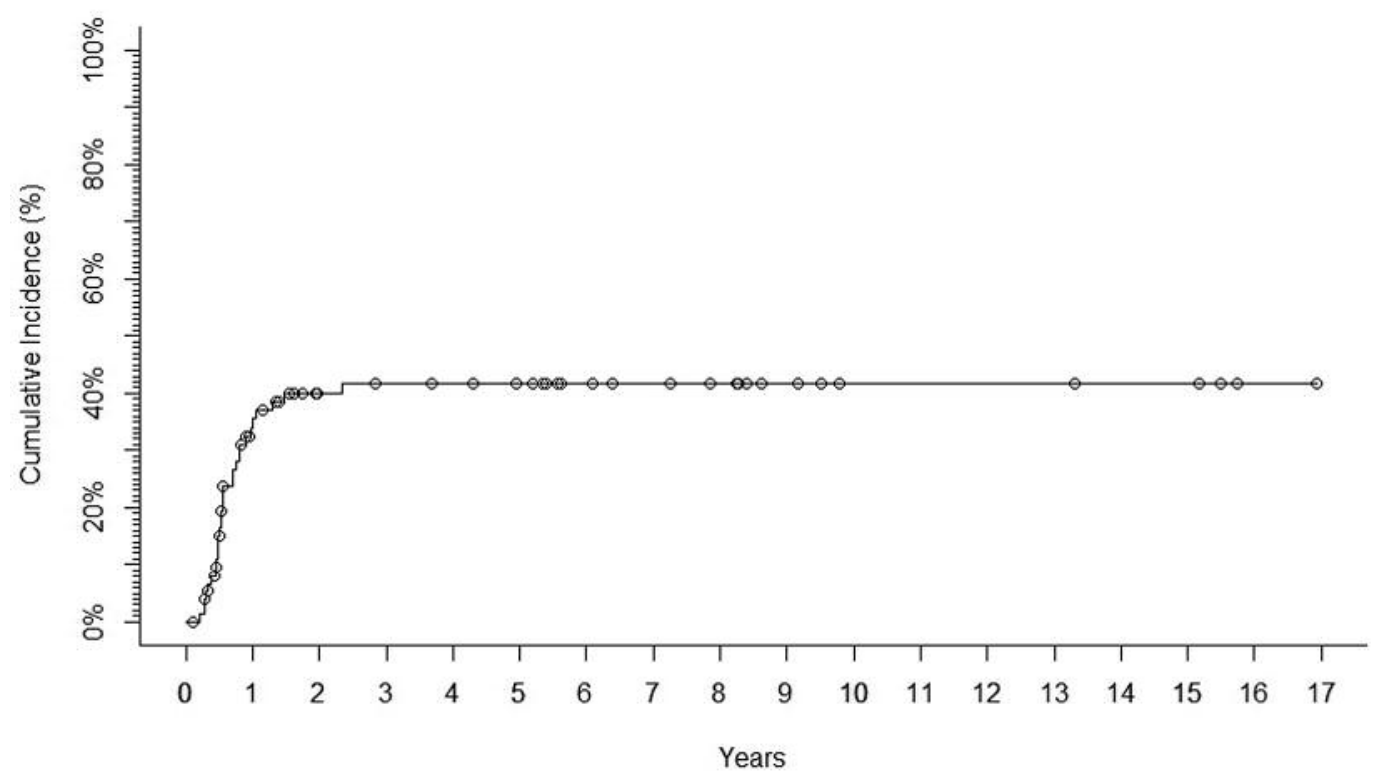

Figure 2. Disease recurrence in 76 patients with node-positive skin cancer.

of cSCC and identified prognostic features of primary tumors to develop an improved staging system, their analysis did not evaluate nodal characteristics, nor did it include immunosuppressed status (6). We retrospectively analyzed
76 patients with cSCC-HN metastatic to regional lymph nodes for risk factors of poor outcomes. We found that an immunosuppressed status was most strongly associated with inferior disease outcomes whereas in immunocompetent 




Figure 3. Disease-free survival, which was statistically different according to immune status.

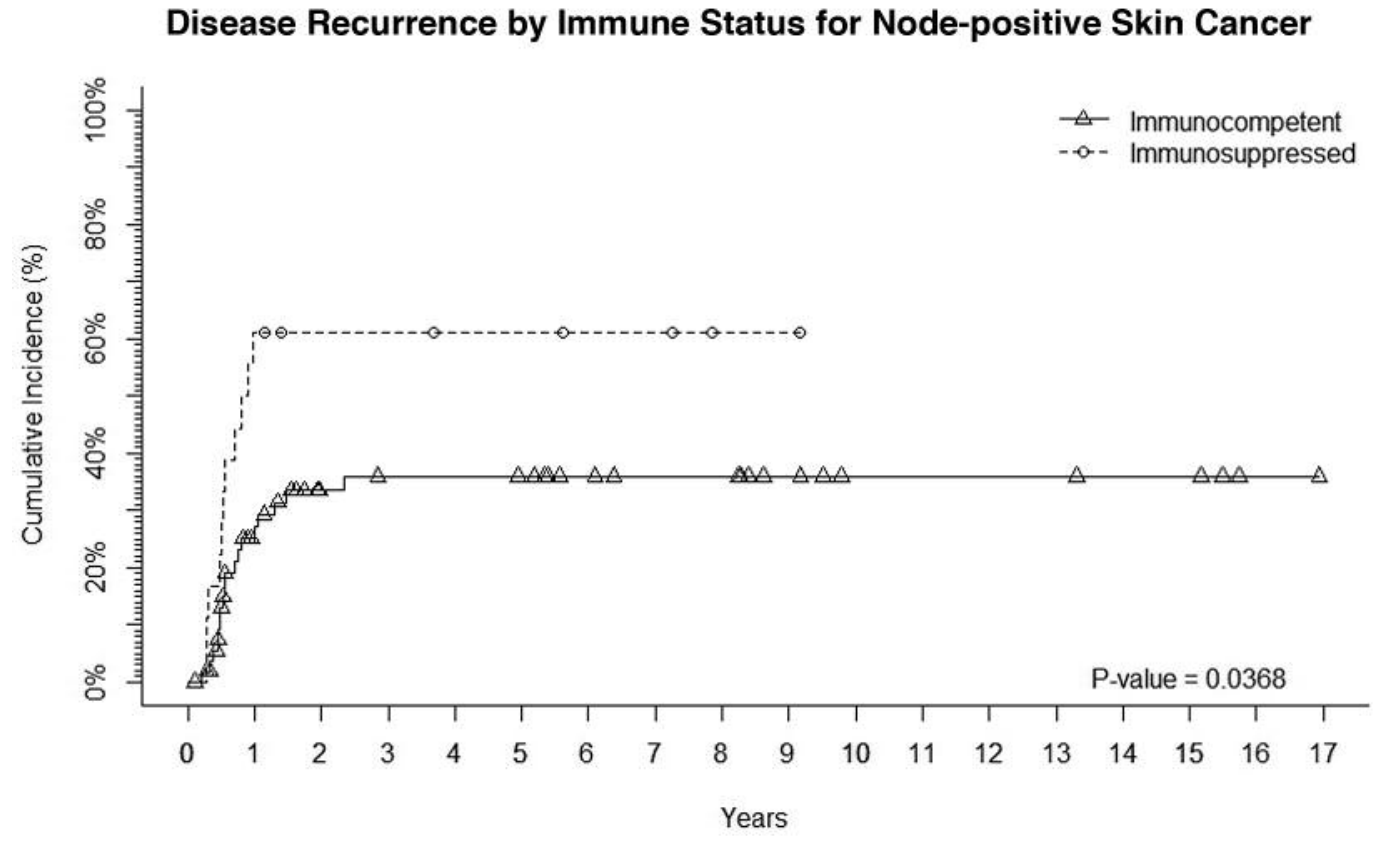

Figure 4. Disease recurrence rates, which were statistically different according to immune status.

patients, ECE was the major driver of disease recurrence. While the use of chemotherapy was also associated with inferior outcomes, this was attributable to our institutional selection bias in which the patients at the highest risk of recurrence were offered systemic therapy.
Patients who are chronically immunosuppressed have a higher incidence of cSCC than the general population and have significantly higher disease recurrence; cSCC is a significant contributor to mortality in these patients reaching up to $5-10 \%$ (7). Other studies have demonstrated that 
Table II. UVA and MVA for variables associated with risk of disease recurrence $(N=76)$.

\begin{tabular}{|c|c|c|c|c|}
\hline \multirow[b]{2}{*}{ Prognostic Factor } & \multicolumn{2}{|c|}{ Univariate analysis } & \multicolumn{2}{|c|}{ Multivariate analysis } \\
\hline & HR $(95 \% \mathrm{CI})$ & $p$-Value & HR $(95 \% \mathrm{CI})$ & $p$-Value \\
\hline Immunosuppressed: Yes vs. no & $2.252(1.06-4.76)$ & 0.0344 & $2.193(1.00-4.81)$ & 0.0498 \\
\hline Chemotherapy: Yes $v s$. no & $2.825(1.37-5.81)$ & 0.0047 & $2.681(1.18-6.10)$ & 0.0187 \\
\hline Cell differentiation: Poor $v s$. well/moderate & $1.601(0.78-3.29)$ & 0.1991 & & \\
\hline ECE: Yes $v s$. no & $5.650(0.70-45.46)$ & 0.1035 & & \\
\hline PNI: Yes vs. no & $1.230(0.60-2.52)$ & 0.5713 & & \\
\hline Tumor classification: $\mathrm{T} 4 v s . \mathrm{Tx}-\mathrm{T} 2$ & $1.592(0.54-4.68)$ & 0.3978 & & \\
\hline Node classification: N2b-N3 vs. N1-N2a & $1.692(0.76-3.79)$ & 0.2 & & \\
\hline
\end{tabular}

Table III. Univariate analysis for disease recurrence in immunocompetent patients only $(N=57)$.

\begin{tabular}{llc}
\hline Prognostic Factor & HR $(95 \% \mathrm{CI})$ & $p$-Value \\
\hline Cell Differentiation: Poor $v s$. well/moderate & $1.824(0.74-4.50)$ & 0.1918 \\
PNI: Yes $v s$. no & $1.462(0.60-3.60)$ & 0.4069 \\
Node classification: N2b-N3 vs. N1-N2a & $2.584(0.87-7.75)$ & 0.0885 \\
Chemotherapy: Yes $v s$. no & $5.155(2.13-12.50)$ & 0.0003 \\
\hline
\end{tabular}

immunosuppression is a significant predictor of poor outcome in cSCC specifically with nodal involvement (10-12). Our results are consistent with previous findings, demonstrating that immunosuppression is a significant risk factor that portends poor prognosis in cSCC. For immunosuppressed patients with nodal spread, who carry a very poor prognosis, clinical trials are urgently needed to help identify intensification strategies that can improve outcomes. The most exciting candidate therapies of this disease are checkpoint inhibitors, specifically those that target the programmed death receptor pathway. A recent phase II study of REGN-2810 (NCT 02760498 ) found a $46 \%$ response rate in patients with metastatic cSCC, many of which were durable. This is the most active compound studied to date and incorporating immunotherapy into the up-front management of these highrisk patients is an attractive clinical question. At Cleveland Clinic, a phase II study of post-operative RT plus concurrent and adjuvant pembrolizumab is being performed for patients with high-risk cSCC-HN. Patients with nodal disease are eligible and the study is open to both immunocompetent patients as well as patients immunosuppressed due to CLL. Patients with iatrogenic immunosuppression for transplants or rheumatic disease are excluded due to the risk of immunotherapy inducing rejection organ and/or exacerbation of their underlying medical condition (NCT 03057613).

Our finding that ECE was highly prognostic in this population is consistent with several other studies $(4,11,13$, 14). In their proposed revision of the clinical nodal staging of cSCC, Forest et al. found ECE to be a significant predictor of survival on univariate analysis, but it did not remain predictive on multivariate analysis (15). Since ECE is positively correlated with lymph node size, studies that concurrently assess both variables may not find ECE to be a statistically significant risk factor (15). A recent randomized trial investigating the addition of chemotherapy to postoperative radiation in immunocompetent patients with high risk cutaneous SCC found that ECE and lymph nodes larger than $2 \mathrm{~cm}$, conferred a substantially higher risk of inferior DFS (personal communication, Sandro Porccedu MD). While additional work is needed to help clarify whether the degree of ECE matters and whether the number of involved nodes and location of nodes (parotid vs. cervical) can help further to risk stratify patients, we do know that ECE is clearly a driving factor in this disease.

As this study was limited to patients who had nodal involvement almost exclusively as recurrent disease, primary tumor characteristics that are often highly prognostic, including PNI, and poorly differentiated tumors, failed to correlate with outcomes (8). Although primary tumor characteristics are generally highly prognostic in localized disease, they may very well be superseded by nodal factors once nodal disease is present (11). The present analysis did not include some nodal characteristics that have been shown to be significant in other studies, such as size of the largest involved lymph node, number of involved lymph nodes, and total lymph node involved ratio, due to insufficient data in older pathology reports (15-17). The best data on outcomes in this disease that was published by the Brigham and 
Women's Hospital group, looked only at primary tumor characteristics, which were incorporated into the $\mathrm{T}$ staging of AJCC 8th edition (6). Future staging studies that can investigate the interactions of $\mathrm{T}$ and $\mathrm{N}$ factors and their impact on outcome are needed to help devise more holistic prognostic classification schemes.

As a retrospective review of a moderate sized cohort, this study is limited by selection bias and limited power to conclusively answer our hypothesis. Also, patients treated in the earlier era of this study had sparse pathologic reports and recall bias, and missing data increased our uncertainty. By limiting this study to a pure population of node-positive cSCC-HN, we believe our findings reflect what we encounter in clinical practice and lends support to the hypothesis that patients with immunosuppression and/or ECE have suboptimal outcomes. Clinical trials investigating treatment intensification are warranted.

\section{Conclusion}

Patients with cutaneous squamous cell carcinoma metastatic to the neck treated with surgery and post-operative radiotherapy, especially those who are immunosuppressed and those with extracapsular nodal extension, have a relatively poor prognosis. Additional studies are needed to incorporate nodal characteristics into prognostic systems and clinical trials are needed to investigate intensification strategies that can improve outcomes.

\section{Conflicts of Interest}

Dr. Brian Gastman: Speaker arrangement with Merck. Dr. Shlomo Koyfman: Research support from Merck. No other Authors have conflicts of interest.

\section{Acknowledgements}

The study was supported by the Melvin Markey Discovery Fund at Cleveland Clinic.

\section{References}

1 Rogers HW, Weinstock MA, Harris AR, Hinckley MR, Feldman $\mathrm{SR}$, Fleischer $\mathrm{AB}$ and Coldiron BM: Incidence estimate of nonmelanoma skin cancer in the United States, 2006. Arch Dermatol 146: 283-287, 2010.

2 Brougham ND, Dennett ER, Cameron R and Tan ST: The incidence of metastasis from cutaneous squamous cell carcinoma and the impact of its risk factors. J Surg Oncol 106: 811-815, 2012.

3 Szewczyk M, Pazdrowski J, Golusinski P, Dańczak-Pazdrowska A, Marszałek S and Golusiński W: Analysis of selected risk factors for nodal metastases in head and neck cutaneous squamous cell carcinoma. Eur Arch Otorhinolaryngol 272: 3007-3012, 2015.

4 Amoils M, Lee CS, Sunwoo J, Aasi SZ, Hara W, Kim J, Sirjani D, Colevas AD, Chang AL and Divi V: Node-positive cutaneous squamous cell carcinoma of the head and neck: Survival, highrisk features, and adjuvant chemoradiotherapy outcomes. Head Neck 39: 881-885, 2017.

5 Ross AS and Schmults CD: Sentinel lymph node biopsy in cutaneous squamous cell carcinoma: a systematic review of the English literature. Dermatol Surg 32: 1309-1321, 2006.

6 Karia PS, Jambusaria-Pahlajani A, Harrington DP, Murphy GF, Qureshi AA and Schmults CD: Evaluation of American Joint Committee on Cancer, International Union Against Cancer, and Brigham and Women's Hospital tumor staging for cutaneous squamous cell carcinoma. J Clin Oncol 32: 327-334, 2014.

7 Koyfman SA, Cooper JS, Beitler JJ, Busse PM, Jones CU, McDonald MW, Quon H, Ridge JA, Saba NF, Salama JK, Siddiqui F, Smith RV, Worden F, Yao M and Yom SS: ACR appropriateness criteria((R)) aggressive nonmelanomatous skin cancer of the head and neck. Head Neck 38: 175-182, 2016.

8 Rowe DE, Carroll RJ and Day CL Jr.: Prognostic factors for local recurrence, metastasis, and survival rates in squamous cell carcinoma of the skin, ear, and lip. Implications for treatment modality selection. J Am Acad Dermatol 26: 976-990, 1992.

9 Amin MB, American Joint Committee on Cancer., American Cancer Society. AJCC cancer staging manual.

10 Schmidt C, Martin JM, Khoo E, Plank A and Grigg R: Outcomes of nodal metastatic cutaneous squamous cell carcinoma of the head and neck treated in a regional center. Head Neck 37: 1808-1815, 2015.

11 McLean T, Brunner M, Ebrahimi A, Gao K, Ch'ng S, Veness MJ and Clark JR: Concurrent primary and metastatic cutaneous head and neck squamous cell carcinoma: Analysis of prognostic factors. Head Neck 35: 1144-1148, 2013.

12 Wang JT, Palme CE, Morgan GJ, Gebski V, Wang AY and Veness MJ: Predictors of outcome in patients with metastatic cutaneous head and neck squamous cell carcinoma involving cervical lymph nodes: Improved survival with the addition of adjuvant radiotherapy. Head Neck 34: 1524-1528, 2012.

13 Oddone N, Morgan GJ, Palme CE, Perera L, Shannon J, Wong E, Gebski V and Veness MJ: Metastatic cutaneous squamous cell carcinoma of the head and neck: the Immunosuppression, Treatment, Extranodal spread, and Margin status (ITEM) prognostic score to predict outcome and the need to improve survival. Cancer 115: 1883-1891, 2009.

14 Joseph MG, Zulueta WP and Kennedy PJ: Squamous cell carcinoma of the skin of the trunk and limbs: the incidence of metastases and their outcome. Aust N Z J Surg 62: 697-701, 1992.

15 Forest VI, Clark JJ, Veness MJ and Milross C: N1S3: a revised staging system for head and neck cutaneous squamous cell carcinoma with lymph node metastases: results of 2 Australian Cancer Centers. Cancer 116: 1298-1304, 2010.

16 Hirshoren N, Danne J, Dixon BJ, Magarey M, Kleid S, Webb A, Tiong A, Corry $\mathrm{J}$ and Gyorki D: Prognostic markers in metastatic cutaneous squamous cell carcinoma of the head and neck. Head Neck 39: 772-778, 2017.

17 Tseros EA, Gebski V, Morgan GJ and Veness MJ: Prognostic significance of lymph node ratio in metastatic cutaneous squamous cell carcinoma of the head and neck. Ann Surg Oncol 23: 1693-1698, 2016. 